\title{
MONISM AND INTRINSICALITY
}

\author{
Kelly Trogdon
}

\begin{abstract}
Central to the programme of sparse ontology is a hierarchical view of reality; the basic entities form the sparse structure of being, while the derivative entities form the abundant superstructure. Priority pluralism and priority monism are both theses of sparse ontology. Roughly speaking, the priority pluralist claims that wholes and their properties ontologically depend on parts and their properties, while the priority monist claims that it goes the other way around. In this paper I focus on Ted Sider's recent argument that priority monism is probably false because it is incompatible with our best account of intrinsicality. In response I propose an account of intrinsicality that is compatible with both priority monism and priority pluralism. I argue that the account, in addition to having the virtue of being neutral between priority monism and priority pluralism, is independently plausible.
\end{abstract}

\section{Introduction}

Both priority pluralism and priority monism (henceforth pluralism and monism) are theses of sparse ontology. Central to sparse ontology is a hierarchical view of reality; the basic entities form the sparse structure of being, while the derivative entities form the abundant superstructure. Roughly speaking, the pluralist claims that wholes and their properties ontologically depend on parts and their properties, while the monist claims that it goes the other way around. More specifically, the pluralist says that (i) there are mereological atoms; (ii) for any complex object $\mathrm{x}$, $\mathrm{x}$ exists in virtue of the existence of its proper parts; and (iii) the properties of $\mathrm{x}$ are instantiated in virtue of properties and relations x's proper parts instantiate. The monist, on the other hand, says that, for any objects $\mathrm{x}$ and $\mathrm{y}$, if $\mathrm{x}$ is a proper part of $y$, then (i) $x$ exists in virtue of the existence of $y$; and (ii) the properties of $\mathrm{x}$ are instantiated in virtue of the instantiation of $\mathrm{y}$ 's properties. ${ }^{1}$ In this paper I focus on Ted Sider's [2007] claim that monism is probably false because it is incompatible with our best account of

\footnotetext{
${ }^{1}$ What I have presented above as monism and pluralism Jonathan Schaffer [forthcoming] presents as versions of monism and pluralism, what he calls 'droopy' monism and pluralism. For reasons that I do not have the space to address here, I think that the 'droopy' versions of these theses are the most plausible versions, and this is why I present the droopy versions as the versions of monism and pluralism in the main text. Contrast monism and pluralism with what Schaffer [2007a; 2007b; forthcoming; unpublished ms] calls 'existence' monism and pluralism. According to the former, there is only one object, the world, while, according to the latter, there are many objects, but they are all mereological atoms. See Schaffer 2007b for an argument that existence monism is preferable to existence pluralism. Returning to (priority) monism, Schaffer [forthcoming] offers three arguments for the thesis. He argues that common sense (in particular the idea that the many parts
} 
intrinsicality. The notion of intrinsicality is of great philosophical importance because it plays an essential role in the formulation of various philosophical issues, including discussions of intrinsic value, narrow content, genuine change, etc. ${ }^{2}$ Given its importance, if monism indeed conflicts with our best account of intrinsicality, we have a good reason to reject monism. I consider Sider's objection cast in terms of two different accounts of intrinsicality both proposed by David Lewis, one of which Sider accepts in a modified form. I argue that monism may indeed be incompatible with each account. Then I propose an account of intrinsicality that is compatible with both monism and pluralism. I argue that my account, in addition to having the virtue of being neutral between monism and pluralism, is independently plausible. I therefore conclude that the objection from intrinsicality does not undermine monism.

The in-virtue-of relation plays an important role in this paper, as evidenced by my formulations of monism and pluralism. So before we begin, let me briefly say something about this relation. How good a grip do we have on the in-virtue-of relation? Good enough, I maintain, to proceed. Following Witmer, Butchard, and Trogdon [2005], when we ask about the quality of our understanding of a notion, we should distinguish between at least two sorts of questions. First, we may be asking about whether there is a theory of the notion in the offing, a theory about its logical features, its relation to other notions, and so on. I certainly have no account of the invirtue-of relation to offer here, but I can say something about the connection between the in-virtue-of relation, modality, and explanation. It is pretty clear that an individual instantiates a property $\mathrm{P}$ in virtue of property $\mathrm{Q}$ only if the instantiation of $Q$ asymmetrically necessitates the instantiation of P. It seems that mere asymmetric necessitation, however, is not a sufficient condition for $\mathrm{P}$ to be instantiated in virtue of Q. Consider, e.g., the following properties: being spherical and being spherical and being green. The instantiation of the latter conjunctive property asymmetrically necessitates the former property, but intuitively the former is not instantiated in virtue of the latter. I take it that the reason we have this intuition is because we recognize that claims about in-virtue-of relations carry explanatory force, and the instantiation of the latter does not explain the instantiation of the former in the appropriate sense. ${ }^{3}$

Another question we might have in mind is this: to what degree do we have a facility with the notion? Our facility with a notion concerns how confidently we apply it, whether we can reach agreement on its proper use in a reasonable range of cases, whether our judgments involving it are stable

are arbitrary portions of the world), considerations involving quantum entanglement (in particular the idea that the world is one vast entangled system), and the epistemic possibility of gunk (the epistemic possibility of matter every part of which has a proper part) each recommend monism over pluralism. Consideration of these arguments, however, falls outside the scope of this paper.

${ }^{2}$ Consider, e.g., the issue of narrow content. The content of a subject's mental state is narrow only if it supervenes on intrinsic properties instantiated by the subject (or perhaps states of the subject), while the content of the state is broad or wide only if this is not the case. There is, of course, much disagreement about whether there is genuine narrow content, but not (to my knowledge, anyway) about whether an existence condition for such content appeals to intrinsicality.

${ }^{3} \mathrm{I}$ am in agreement with Schaffer [forthcoming] in not being hopeful about the prospects for an analysis of the in-virtue-of relation (what Schaffer calls the relation of 'priority'). Our concept of the in-virtue-of relation resembles most of our interesting philosophical notions in this respect. 
and not subject to inexplicable shifts, and so on. With respect to the matter of our facility with the in-virtue-of notion I think it is safe to say that our grasp of it is fairly secure. Philosophers and non-philosophers alike make frequent use of the 'in virtue of' locution, and there seems to be much agreement about its application in a broad range of uncontroversial cases, e.g. when we talk about rights, responsibilities, and powers one acquires through the social roles one plays, as in 'He has the right to vote in virtue of being a citizen.'

Since we have a facility with the in-virtue-of notion and understand that the in-virtue-of relation at least involves asymmetric necessitation as well as explanation, I conclude the notion is well understood enough for us to proceed. I therefore ask that we put potential concerns about the in-virtueof relation to the side and proceed to the main task of this paper, that of articulating and defending an account of intrinsicality compatible with both pluralism and monism. ${ }^{4}$

\section{The Objection from Intrinsicality}

\section{A. Lewis's First Account}

Sider proposes various objections to monism, one of which involves Lewis's [1983; 1986: 61-2] conception of intrinsic properties. ${ }^{5}$ Lewis's proposal is as follows:

Duplication: Objects are duplicates just in case their parts may be put in oneone correspondence preserving the perfectly natural properties and relations.

Intrinsicality: A property is intrinsic just in case it can never differ between a pair of possible duplicates (it cannot 'divide' duplicates).

Sider's objection from intrinsicality is fairly condensed, and I unpack it thus: (i) a version of Lewis's account of intrinsicality (one Sider proposes that takes into account the epistemic possibility of gunk) is both complete (i.e., for any qualitative property $\mathrm{P}$, the account delivers a verdict on whether $\mathrm{P}$ is intrinsic or non-intrinsic) and probably correct; (ii) this account is incompatible with monism; therefore, (iii) monism is probably false. Sider defends (i) elsewhere (see Sider [1993: Ch. 4] and Sider [1996]), and his argument for (ii) is as follows.

Following Lewis, Sider identifies the perfectly natural properties with the fundamental properties. (A fundamental property, very roughly, is a property which, though itself not instantiated in virtue of other properties,

\footnotetext{
${ }^{4}$ See Witmer, Butchard, and Trogdon [2005] for a more substantial discussion of our facility with the invirtue-of notion and for more on asymmetric necessitation and the in-virtue-of relation.

${ }^{5}$ Sider [2007] casts his objections, including the objection from intrinsicality, as objections to existence monism (see note 1). He claims, however, that his objections to existence monism apply to (priority) monism as well. I think that monism but not existence monism is a live option, so I consider the objection from intrinsicality in terms of the former rather than the latter.
} 
is such that other properties are instantiated ultimately in virtue of it. ${ }^{6}$ ) Sider accordingly concludes that, since the monist claims that the world as a whole rather than any of its proper parts instantiates the fundamental properties, she is committed to the claim that the world as a whole rather than any of its proper parts instantiates the perfectly natural properties. If the monist is committed to this claim, she is thereby committed to the claim that the various denizens of the world, e.g. microscopia like electrons and quarks, ordinary objects like tables and rocks, and human beings as well as other organisms, fail to instantiate any perfectly natural properties. But if these individuals fail to instantiate perfectly natural properties, they have no possible duplicates as characterized above. In this case there are two possible consequences for Lewis's account. First, we might conclude that Lewis's account does not deliver any verdict on which qualitative properties of objects distinct from the world are intrinsic and which ones are non-intrinsic, so it is incomplete in the sense characterized above. Here the idea is that if, e.g., there is no possible duplicate of the coffee cup on the table, the account simply fails to apply to the cup's properties. Second, we might conclude that Lewis's account, though complete, classifies all of the cup's properties as intrinsic, so it is simply incorrect. Here the idea is that no property of the cup can divide its possible duplicates because it has no possible duplicates, so each of its properties trivially meets the condition for being intrinsic. Either way, there is a tension between monism and (i) from above.

\section{B. Lewis's Second Account}

Lewis goes on to propose another account of intrinsicality Sider does not consider, one Lewis prefers to his original account, so we will do well to consider whether it too is incompatible with monism. ${ }^{7}$ Clearly the notion of intrinsicality has something to do with the notion of independence. An intrinsic property, it is said, is had in a way that is independent of the way wholly distinct contingent individuals are. ${ }^{8}$ Moreover, it is thought that whether an individual instantiates an intrinsic property is independent of whether there are any wholly distinct contingent individuals in the first place. Rae Langton and David Lewis [1998] (henceforth L\&L) may have the latter intuition in mind in claiming that intrinsic properties are independent of accompaniment. Let an accompanied individual be one that coexists with

\footnotetext{
${ }^{6}$ This rough gloss of fundamentality, of course, is not intended as a serious account. How we are to precisely characterize fundamentality is a difficult matter. Not being instantiated in virtue of any property is a necessary but not a sufficient condition for being fundamental, given that non-qualitative non-fundamental properties like being identical to Kelly arguably are not instantiated in virtue of any properties. Assuming that there are non-fundamental properties that are not instantiated in virtue of any properties, we need to modify our initial characterizations of monism and pluralism. In this case, clause (iii) of the characterization of pluralism should read 'the properties of $\mathrm{x}$, if instantiated in virtue of any properties, are instantiated in virtue of properties and relations x's proper parts instantiate', and a modification of the same sort is necessary for clause (ii) of the characterization of monism. Thanks to Maya Eddon for helpful discussion on these points.

${ }^{7}$ Lewis claims that his new account is consistent with, but possesses certain theoretical advantages with respect to, the proposal outlined above. He claims, e.g., that the new account does not require any particular way of drawing the distinction between natural and non-natural properties, only that there be some way of drawing the distinction.

${ }^{8}$ Objects $\mathrm{x}$ and $\mathrm{y}$ are wholly distinct from one another just in case they share no parts.
} 
some contingent individual wholly distinct from it and a lonely individual be one that is not accompanied. We can then say that a property $\mathrm{P}$ is independent of accompaniment just in case each of the following four situations is possible: a lonely individual has P; a lonely individual lacks P; an accompanied individual has $\mathrm{P}$; and an accompanied individual lacks $\mathrm{P}$.

L\&L point out, however, that not all properties that are independent of accompaniment are intrinsic. Consider, e.g., the disjunctive property being cubical and lonely or non-cubical and accompanied. This property is intuitively non-intrinsic but nonetheless independent of accompaniment. (It is independent of accompaniment because a lonely individual can have it by being a cube that is lonely, a lonely individual can lack it by being a noncube that is lonely, an accompanied individual can have it by being a noncube and accompanied, and an accompanied individual can lack it by being a cube and accompanied.) L\&L design their account of intrinsicality to appeal to independence of accompaniment but not count this property as intrinsic. First, they define 'disjunctive property' as follows: a property is disjunctive just in case it can be expressed as a disjunction such that the disjuncts are more natural than the disjunction itself. ${ }^{9}$ Then they propose their account of intrinsicality:

Basic intrinsicality: $\mathrm{P}$ is a basic intrinsic property just in case (i) $\mathrm{P}$ is independent of accompaniment; and (ii) $\mathrm{P}$ is neither a disjunction nor a negation of a disjunction.

Intrinsicality: $\mathrm{P}$ is an intrinsic property just in case either (i) $\mathrm{P}$ is a basic intrinsic property; or (ii) $\mathrm{P}$ is definable using basic intrinsic properties and truth-functional compounds thereof.

The disjunctive property in question, though independent of accompaniment, is not counted as intrinsic by this account because it violates clause (ii) of Intrinsicality.

Is monism compatible with this account of intrinsicality? I will argue that it is not. I present the argument in two stages. Following Josh Parsons [2004], being polka-dotted and being hot at one end and cold at the other are examples of what he calls 'distributional properties'. Intuitively, a distributional property is a way of filling in a spatially-extended object with some property such as colour, heat, density, and so on. Call the properties that are filled in 'distributable' properties. Parsons argues, to my mind persuasively, that distributional properties are something over and above structural properties, i.e. properties that are analysable solely in terms of non-distributional properties. ${ }^{10}$ So the distributional property being red

\footnotetext{
${ }^{9} \mathrm{~L} \& \mathrm{~L}$ actually stay neutral on whether naturalness is a matter of degree; they claim that if it is not a matter of degree, then a property is disjunctive just in case it can be expressed as a disjunction such that the disjuncts are natural properties. Since Lewis's original account of intrinsicality is cast in terms of degreed naturalness (recall the talk of 'perfectly' natural properties), I will continue to work with this notion. Nothing I say in this paper, however, hinges on interpreting naturalness as coming in degrees.

${ }^{10}$ One of Parsons's arguments, very roughly, is this. First, suppose that gunk is metaphysically possible. Second, suppose that an object $\mathrm{x}$ is a piece of gunk and $\mathrm{x}$ instantiates a distributional property $\mathrm{P}$. In this case it is metaphysically possible that every proper part of $\mathrm{x}$ instantiates distributional properties in such a way that there is no non-distributional ground for $\mathrm{P}$. We will return to this argument in note 21 .
} 
polka-dotted on an otherwise white background, e.g., is something over and above any structural property analysable in terms of redness and whiteness, understood as non-distributional properties, and various distance relations. Getting back to monism, let us suppose that the monist claims that the world instantiates one big distributional property, D, the property tracing such-and-such a path through physical configuration space, and D is fundamental. ${ }^{11}$ In the first stage of the argument for the claim that monism and L\&L's account are incompatible, I argue that if monism is true and thus $\mathrm{D}$ is fundamental, D is intrinsic as well. In the second stage I argue that if monism is true, L\&L's account says that D is non-intrinsic.

If monism is true and D, a global distributional property, is the one and only fundamental property, why think that it is intrinsic? ${ }^{12}$ One might argue that reflection on physical theory tells against the idea that fundamental properties must be intrinsic. Steve Yablo [1998], e.g., argues that there is no principled reason why theorists of the quantum domain should not find themselves forced by the phenomenon of quantum entanglement to count certain non-intrinsic properties as fundamental, and there is nothing in the nature of intrinsicality to prevent this. An example of two entangled quantum systems is this: two particles such that when one is observed to be spin-up, the other will always be observed to be spin-down and vice versa, despite the fact that it is impossible to predict, according to quantum mechanics, which set of measurements will be observed. As a result, measurements performed on one system seem to be instantaneously influencing the other system entangled with it. ${ }^{13}$

I think, pace Yablo, that there is something about the nature of intrinsicality that may preclude non-intrinsic properties from being fundamental. The idea is straightforward. If $\mathrm{P}$ is a fundamental property, $\mathrm{P}$ is not instantiated in virtue of the instantiation of any other property. From this it trivially follows that $\mathrm{P}$ is not instantiated in virtue of how any wholly distinct (contingent) object is. Hence $\mathrm{P}$, qua fundamental property, conforms to a condition that is constitutive of one of our central intuitions about intrinsicality: an intrinsic property is one such that an object having it does not depend on how other (contingent) objects are. So given that fundamental properties are not instantiated in virtue of the instantiation of other properties, their instantiation does not depend on the instantiation of other properties (of wholly distinct contingent objects), so it seems that they are not non-intrinsic.

\footnotetext{
${ }^{11}$ Schaffer [forthcoming] considers the property mentioned above, and whether it, qua distributional property in Parsons's sense, is fundamental. As an advocate of monism, however, Schaffer officially stays neutral on this issue.

${ }^{12}$ We should note that there is an esteemed tradition, including the likes of Leibniz, Kant, and Russell (culminating with Lewis), sympathetic with the claim that non-intrinsic properties must be instantiated ultimately in virtue of intrinsic properties. Leibniz, e.g., argues that no possible substantial entity instantiates only extrinsic properties; 'there is no denomination so extrinsic that it does not have an intrinsic denomination at its basis. This is itself one of my important doctrines (kyriai doxai)' [1975 (1702), quoted in Pereboom unpublished $\mathrm{ms}]$.

${ }^{13}$ Gene Witmer [personal communication] has suggested that we need not appeal to curious discoveries in quantum mechanics to show that fundamental non-intrinsic properties are at least epistemically possible. Suppose that electrons, protons, and neutrons, as in the familiar simple theory of the atom, really are mereological atoms. Suppose further that some particular electron has the non-intrinsic property of being in orbit about some neutron/proton cluster. The idea is that the world might turn out such that this property is fundamental.
} 
To say that fundamental properties match our intuitions about one aspect of intrinsicality is a good start, but it does not constitute a substantial argument for the claim that fundamental properties are intrinsic. Let me therefore change tactics and instead debunk one potential motivation for thinking that at least some fundamental properties are non-intrinsic. One might have in mind the idea that relational non-intrinsic properties could turn out to be fundamental. Suppose that pluralism is true. Consider those relations instantiated by mereological atoms that are not instantiated in virtue of the intrinsic properties of the atoms themselves. Are these relations not fundamental and non-intrinsic? Well, following Lewis [1986: 62], we can say the following. Consider the (arguably) fundamental relation of distance. Suppose that the distance between mereological atoms $\mathrm{x}$ and $\mathrm{y}$ is $\mathrm{R}$. Though $\mathrm{R}$ is not instantiated in virtue of any of the properties of $\mathrm{x}$ and $\mathrm{y}$ taken individually, $\mathrm{R}$ is instantiated in virtue of the intrinsic properties of the fusion of $\mathrm{x}$ and $\mathrm{y}$. In Lewis's terminology, distance is an 'external relation', and external relations (according to Lewis, anyway) are intrinsic. In this case the pluralist can claim that the fundamental level of reality consists of mereological atoms as well as certain fusions of them, and the fundamental properties and relations are instantiated by the occupants of this level. ${ }^{14,15}$

Now we can turn to the second stage of the argument. Here I argue that if monism is true then L\&L's account of intrinsicality counts D as a nonintrinsic property. As we have already discussed, fundamental properties are not instantiated in virtue of any other properties. I think, moreover, that it is impossible for them to be instantiated in this way. Here I follow Lewis [1986: 60 , note 44] in claiming that $\mathrm{P}$ is a fundamental ('sparse' as opposed to 'abundant') property only if it is necessary that, for any object $\mathrm{x}$, if $\mathrm{x}$ has $\mathrm{P}$, then there is no object $y$ and property $Q$ such that $x$ has $P$ in virtue of $y$ 's having Q. Here the idea is that fundamentality is absolute rather than worldrelative; fundamental properties are essentially fundamental. The essentiality of fundamentality plays an important role in the second stage of my argument, so let me say something in defence of it.

Why think that fundamentality is absolute? Jonathan Schaffer [personal communication] claims that the following consideration might support the idea that fundamentality is instead world-relative. Suppose, as I think is quite plausible, that what Schaffer [2005] calls 'quiddistic contingentism', the claim that the laws of nature are contingent by virtue of the fact that there are counterfactual possible worlds in which properties instantiated in the actual world disassociate from their actual nomic roles and causal powers, is correct. Schaffer claims that, if a property's being fundamental (in a world) is connected to its nomic role and causal powers (in that world), then, if

\footnotetext{
${ }^{14}$ I will have more to say about levels of reality later in the paper.

${ }^{15}$ One might argue that certain vector properties, e.g. having velocity $v$ (where $\mathrm{v}$ is some vector) are both fundamental and non-intrinsic. I do not have the space here to pursue this issue in any detail, but I can say that I think this is a difficult matter and that it is certainly not obvious that fundamental vector properties are non-intrinsic. As evidence of this, recall Brian Weatherson's [2006] interesting discussion of what he calls the 'asymmetric magnets problem'. His response to the problem is nuanced: he claims that we are to conclude that fundamental vector features are intrinsic in one important sense (they conform to a modified version of Lewis' duplication thesis) but not in another (changing their direction does not change the intuitively intrinsic properties of objects).
} 
quiddistic contingentism is true, it would seem that fundamentality is worldrelative.

I think of fundamentality, however, somewhat differently. In particular, I distinguish between the causal/nomic profile of a property $\mathrm{P}$ and what I call P's 'in-virtue-of profile' (the facts about which properties $\mathrm{P}$ can be instantiated in virtue of, and which properties can be instantiated in virtue of $\mathrm{P}$ ) and claim that the latter but not the former is central to fundamentality. The idea is that, though the causal/nomic profile of $\mathrm{P}$, be $P$ fundamental or non-fundamental, varies across possible worlds depending on the laws of nature, certain in-virtue-of facts hold constant across the space of possible worlds, including facts like ' $P$, if instantiated, is not instantiated in virtue of the instantiation of $Q$,' etc. If $P$ is fundamental, then one fact that holds constant, I claim, is ' $\mathrm{P}$, if instantiated, is not instantiated in virtue of the instantiation of any other property'. My claim, then, is that certain aspects of the in-virtue-of profile of a property are tied to its essence, while no aspect of its causal/nomic profile is so tied. ${ }^{16}$

Why think that properties have something like in-virtue-of profiles as described above that render fundamentality absolute? Well, let us agree to take seriously the idea of objective similarity between individuals. Since objective similarity is not a world-relative notion, then, if (as I am inclined to think) sameness in fundamental properties makes for objective similarity, ${ }^{17}$ we should expect that fundamentality is not world-relative either. ${ }^{18}$ Here is a sketch of the idea I have in mind. Suppose that $\mathrm{x}$ in @ (the actual world) objectively resembles y in some merely possible world w. Suppose further that the causal/ nomic profiles of the fundamental properties in @ and those in w are quite different. (At this point we are staying neutral on whether this shows that the fundamental properties instantiated in @ and w are distinct.) On the assumption that sameness in fundamental properties underwrites objective similarity, we can conclude that $\mathrm{x}$ and $\mathrm{y}$ share some property $\mathrm{P}$ that is fundamentalin@ as well as in w. Given that the fundamental properties in @ and $\mathrm{w}$ have different causal/nomic profiles, the notions of fundamentality and causal/nomic profile therefore come apart. Hence, something besides the causal/nomic profile of $\mathrm{P}$ must underwrite the fundamentality of $\mathrm{P}$. The claim that the fundamentality of a property is determined by its (essential) in-virtueof profile (or essential aspects thereof) is a natural candidate.

Ben Caplan [personal communication], however, has posed the following objection to the claim that, on the monist framework, D from above is essentially fundamental. Following Peter Vallentyne, a contraction of a world is "a world "obtainable" from the original one solely by "removing" objects from it' [1997: 211]. Consider a world w that is a contraction of the actualworld@ that lacks one of its proper parts, say my left shoe. (As a contraction of @, w is otherwise as similar to @ as possible.) Let us assume with Schaffer [forthcoming] that monism, if true, is necessarily

\footnotetext{
${ }^{16}$ Recall my earlier claim that there is an explanatory component to the in-virtue-of relation. If the actual fundamental properties can disassociate from their actual causal/nomic profiles, then it seems to follow that the sense in which the instantiation of fundamental properties explains the instantiation of non-fundamental properties is ultimately neither causal nor nomic.

${ }^{17}$ See Lewis [1983] for a seminal discussion of these and related issues

${ }^{18}$ Thanks to Phil Bricker for suggesting this idea.
} 
true. ${ }^{19}$ Hence, assuming that monism is true of @, it is true of w as well. Let $\mathrm{D}^{*}$ be the global distributional property that is fundamental in w. Caplan suggests that it seems that $\mathrm{D}^{*}$ is instantiated in @ as well, and $\mathrm{D}^{*}$ is instantiated in virtue of the instantiation of D in @. But if this is right, then $\mathrm{D}^{*}$ is fundamental in one world (w) but not in another (@), so fundamental properties are not essentially fundamental.

How should we respond to this objection? Here is my proposal. First, global distributional properties stand in the determinable-determinate relation. ${ }^{20}$ Just as the property having a colour, qua determinable, is general in nature, while being red, qua determinate of having a colour, is more specific, some global distributional properties, qua determinables, are general in nature, while others, qua determinates of those determinables, are more specific. The maximally specific global distributional properties completely specify the mereological structure of worlds that instantiate them while their determinables do not. If we treat the global distributional property of a world that completely specifies its mereological structure as the fundamental property of that world, then we have a response to Caplan's objection. Given that the mereological structure of @ andwdiffer (one has my left shoe as a proper part and the other does not), they do not instantiate the same fundamental property. They will have many global distributional properties in common (properties that are not maximally specific regarding their respective mereological structures), but these properties are non-fundamental. The overall idea, then, is that $\mathrm{D}^{*}$ is not instantiated in@, given that $\mathrm{D}^{*}$ provides a complete specification of a mereological structure different from the one embodied in @.21

Now let us finally complete the second stage of the argument. If monism is true, then D is a perfectly natural property, so, by L\&L's lights, it is neither a disjunction nor a negation of a disjunction, for it cannot be expressed as a disjunction or a negation of a disjunction such that its disjuncts are more natural than itself because there are no properties more natural than D. So far so good. But is D independent of accompaniment? D is independent of accompaniment just in case the following situations are possible: a lonely individual has D; a lonely individual lacks D; an accompanied individual has $\mathrm{D}$; and an accompanied individual lacks $\mathrm{D}$. The actual world is lonely

\footnotetext{
${ }^{19}$ Recall (see note 1) that what I am calling 'monism' and 'pluralism' Schaffer presents as versions of monism and pluralism, what he calls 'droopy' monism and pluralism. In Schaffer [forthcoming], he claims only that monism (pluralism) is if true necessarily true - he does not make this claim specifically about droopy monism (pluralism). In conversation, however, Schaffer has informed me that he is inclined to accept the further claim. ${ }^{20}$ Thanks to Maya Eddon for helpful discussion on this response to Caplan. Parsons [2004] claims that distributional properties stand in the determinable-determinate relation, though in his discussion he focuses on nonglobal distributional properties. (He claims, for example, that the property having a colour distribution is a determinable, and being polka-dotted is one of its determinates, as is being uniformly red all over. Schaffer [forthcoming] claims that global properties stand in the determinable-determinate relation, though he does not say this specifically about global distributional properties. His example is the determinable global property being heterogeneous and the determinate global property tracing such-and-such a path through physical configuration space. ${ }^{21}$ In his argument for the claim that distributional properties are something over and above structural properties, Parsons [2004] seems to assume that the very same distributional properties can be instantiated by pieces of gunk as well as objects consisting of mereological atoms. Parsons's argument, if successful, therefore, only shows that global distributional properties that are general in nature (ones that do not completely specify the mereological structure of the worlds that instantiate them) are something over and above structural properties. On the face of it, methodological principles concerning simplicity and uniformity, however, recommend that if some distributional properties are something over and above structural properties, then all such properties are something over and above structural properties.
} 
and, we are assuming, has D, so the first condition is met. ${ }^{22}$ There is a possible (lonely) counterfactual world, one of whose proper parts is qualitatively distinguishable from a proper part of the actual world, so this counterfactual world lacks D; thus the second condition is met as well. Further, there are possible worlds with proper parts that lack D (the actual world is such a world) so there are possible accompanied individuals that lack D; hence the fourth condition is also satisfied. Things are not so clear, however, with the third condition. It is possible for an accompanied individual to have $\mathrm{D}$ if there is a counterfactual possible world, w, one of whose proper parts has D (you can think of $w$ as an expansion of the actual world in the sense that it contains the actual world as a proper part). It would seem, however, that $\mathrm{w}$ is impossible, for recall that, on our account of fundamentality, if D is fundamental, D is essentially fundamental in the sense that there is no possible world in which an object has D in virtue of an object's having some other property. Assuming that monism, if true, is necessarily true, then every possible world is such that if a proper part of that world has some property, that part has that property in virtue of some property or properties the world as a whole instantiates. Hence, $w$ is impossible because one of its proper parts instantiates a property (D) that is not instantiated in virtue of the world's instantiating some property. Assuming, as seems plausible, that the possibility of worlds like w (worlds such that their proper parts instantiate fundamental properties) is not only sufficient but necessary for $\mathrm{D}$ to meet the third condition, then, given that such worlds are impossible on monism, D is not independent of accompaniment on monism. If $\mathrm{D}$ is not independent of accompaniment, L\&L's account of intrinsicality says that D is not a basic intrinsic property. Moreover, if $\mathrm{D}$ is not independent of accompaniment, $\mathrm{D}$ is not a non-basic intrinsic property either, for if $\mathrm{D}$ were definable using properties that are themselves independent of accompaniment and truthfunctional compounds thereof, surely D would be independent of accompaniment as well. ${ }^{23}$ I conclude that monism is incompatible with L\&L's account.

\section{Intrinsicality for Monists and Pluralists}

\section{A. The In-virtue-of Account}

I am suspicious of the idea of defining intrinsicality in terms of naturalness, so I am not sympathetic with either of the accounts of intrinsicality discussed above anyway. ${ }^{24}$ Moreover, I see the dialectic with respect to

\footnotetext{
${ }^{22}$ One might claim that a lonely object cannot have $\mathrm{D}$ because the configuration space of a physical system is defined mathematically, and numbers are wholly distinct from any physical system. But remember that the notion of distinctness at issue with independence of accompaniment is distinctness from contingent individuals. Hence, assuming that numbers are necessary existents, properties that are defined mathematically do not thereby violate the first condition for independence of accompaniment.

${ }^{23}$ If pluralism instead of monism is true, then, since D is not a fundamental property, it meets all four conditions for independence of accompaniment. If $\mathrm{D}$ is definable using basic intrinsic properties and truthfunctional compounds thereof, L\&L's account will classify D as intrinsic.

${ }^{24}$ See Witmer, Butchard, and Trogdon [2005] for a discussion of why it is a bad idea to define intrinsicality in terms of naturalness. In a later paper, 'Redefining Intrinsic' [2001] Lewis advances yet another account of intrinsicality, but it too appeals to naturalness so I will not consider it here.
} 
pluralism, monism, and intrinsicality somewhat differently from Sider. For me, pluralism and monism are each epistemic possibilities, so it is a virtue of an account of intrinsicality to be neutral between them. Let us see, then, if we can set forth a plausible account of intrinsicality that is neutral between pluralism and monism.

In Witmer, Butchard, and Trogdon [2005] we offer an account of intrinsicality that appeals to the notion of independence of accompaniment but not naturalness, so let us consider whether monism in addition to pluralism is compatible with our account. To set the stage for the account, let us return to the disjunctive property discussed earlier. Why do we all find it intuitive to say that being cubical and lonely or non-cubical and accompanied is non-intrinsic? Well, suppose that some individual has this property, and it has the property because it is cubical (presumably an intrinsic property) and lonely (clearly a non-intrinsic property). Hence, on this occasion its having this disjunctive property depends on its having certain non-intrinsic properties. It is, we maintain, the fact that it is so immediately obvious that an individual can have the disjunctive property in virtue of its having certain plainly non-intrinsic properties that drives us to classify the disjunctive property itself as non-intrinsic. The moral we draw is this: if a property $\mathrm{P}$ is such that a possible object instantiates $\mathrm{P}$ in virtue of some non-intrinsic property Q, P itself is non-intrinsic. Consider, then, the following rough, circular characterization of intrinsicality: intrinsic properties are independent of accompaniment, and they cannot be instantiated in virtue of non-intrinsic properties. To remove the circularity inherent here, we propose to discharge the notion of being non-intrinsic from the characterization and replace it with the notion of independence of accompaniment, given that all intrinsic properties are independent of accompaniment. We arrive at the following account of intrinsicality:

Intrinsic fashion: $\mathrm{x}$ has $\mathrm{P}$ in an intrinsic fashion just in case (i) $\mathrm{P}$ is independent of accompaniment; and (ii) for any individual $\mathrm{y}$ and property $\mathrm{Q}$, if $\mathrm{x}$ has $\mathrm{P}$ in virtue of y's having $\mathrm{Q}, \mathrm{Q}$ is also independent of accompaniment.

Intrinsicality: Property $\mathrm{P}$ is intrinsic just in case, for any possible individual $\mathrm{x}$, if $\mathrm{x}$ has $\mathrm{P}, \mathrm{x}$ has $\mathrm{P}$ in an intrinsic fashion.

Call this the 'in-virtue-of account' of intrinsicality. ${ }^{25}$ The core insight behind this account is the idea that an intrinsic property by its nature meets two conditions. First, it is independent of accompaniment. Second, if it is possible for the property to be instantiated in virtue of some further property, this further property itself is independent of accompaniment. Non-intrinsic properties, on the other hand, by their nature either are not independent of accompaniment, or possibly instantiated in virtue of properties that are not themselves independent of accompaniment.

\footnotetext{
${ }^{25}$ Earlier I noted that we have a facility regarding the in-virtue-of notion. But the same is true of the notion of intrinsicality; we confidently apply it, can reach agreement on its proper use in a reasonable range of cases, and so on. In presenting the in-virtue-of account of intrinsicality, however, the idea is that we understand the in-virtue-of notion better than we understand that of intrinsicality.
} 
Let us turn to its application to get a better handle on the account. To begin, we return to the disjunctive property discussed above. Our account easily handles this property, for it is not necessary that, for any individual $y$ and property $\mathrm{P}$, if $\mathrm{x}$ has the disjunctive property in virtue of $\mathrm{y}$ 's having $\mathrm{P}$, then $\mathrm{P}$ is independent of accompaniment. $\mathrm{P}$, e.g., might be being a lonely cube, which is not independent of accompaniment.

Second, let us turn to two properties Brian Weatherson [2001] discusses: being the only round thing and being one of at most 17 cubes. Assuming these properties are indeed non-intrinsic, an adequacy condition for our account is that there are possible cases in which these properties are instantiated in virtue of properties that are themselves not independent of accompaniment. Such cases are not hard to imagine: the former can be instantiated in virtue of being a lonely round thing, and the latter can be instantiated in virtue of being one of exactly 15 cubes.

Third, consider Ted Sider's [2001] example of the 'border-sensitive' property being a rock. Though this property is independent of accompaniment, whether something is a rock depends on what surrounds it, so intuitively it is non-intrinsic. Does our account classify the property as nonintrinsic? Well, imagine an individual that is a piece of sandstone lying in a field of grass. We propose that the individual is a rock in virtue of instantiating (among other properties) being embedded in an environment dissimilar to sandstone, and this property is clearly not independent of accompaniment. Hence, the account gets what is the intuitively right result (at least after we internalize Sider's discussion of border-sensitive properties), that being a rock is non-intrinsic.

To fully motivate our account, I would need to consider various possible objections to it. Though I do not have the space to do this here, ${ }^{26}$ I will consider one objection the response to which highlights an important feature of our account. Brian Weatherson [personal communication] has proposed an interesting objection to our treatment of being a rock. Let $\mathrm{A}$ be the piece of sandstone in the field from above, and let $\mathrm{B}$ be an intrinsic duplicate rock that is in a void, i.e. surrounded by nothing. Weatherson suggests that we are committed to the claim that A is a rock in virtue of very different properties than B, given that B is not embedded in something that differs from sandstone, since it is not embedded in anything at all. Weatherson claims that this seems wrong, for we do not want to say that what makes it the case that $\mathrm{A}$ is a rock is very different from what makes it the case that B is a rock. He claims that the natural thing to say is that something is a rock in virtue of having the right intrinsic properties and not being embedded in a sufficiently similar environment. This is a property that A and B share, but, unfortunately for us, it is independent of accompaniment.

The thing to say in response to Weatherson's objection is that we can accept multiple, overlapping grounding properties. We are not, therefore, forced to choose between saying that the individual in the field is a rock in

\footnotetext{
${ }^{26}$ In Witmer, Butchard, and Trogdon [2005] we consider and respond to various possible objections to the account. We note that our account applies to neither indiscriminately essential properties (e.g. being selfidentical) nor impure properties (being identical to Kelly). We argue [2005: 347ff] that restricting our account of intrinsicality in this way is well motivated.
} 
virtue of either being embedded in an environment that differs from sandstone or in virtue of not being embedded in a sufficiently similar environment; we can say that it is a rock in virtue of having both properties. Hence, it is unclear that we are committed to the claim that what makes the individual in the field a rock is quite different from what makes the individual in the vacuum a rock, for we can say that these individuals qua rocks share some grounding properties but not others. In order for our account to classify being a rock as non-intrinsic, it need not be the case that the property is always instantiated in virtue of being embedded in an environment dissimilar to sandstone, but only that it is possibly instantiated in this way.

Now that we have some idea of how the in-virtue-of account works and why it may be plausible, suppose for the moment that it is correct. We offered this account with something like pluralism as a background assumption, so let us see how things play out if we assume monism instead. If monism is true, then, for any property $\mathrm{P}$, if $\mathrm{P}$ is instantiated by a proper part of the actual world, $\mathrm{P}$ is instantiated in virtue of the world as a whole instantiating D. Since D is not independent of accompaniment, the invirtue-of account says that any such $\mathrm{P}$ if instantiated in the actual world is instantiated in a non-intrinsic fashion, so it is a non-intrinsic property. In this case if monism is true then not only is D non-intrinsic, but also any property instantiated by any proper part of the actual world is non-intrinsic. Indeed, assuming that monism, if true, is necessarily true, then any property instantiated by any proper or improper part of any possible world is nonintrinsic. Assuming that all properties are possibly instantiated, it follows that all properties are non-intrinsic. If pluralism instead of monism is true, then it is not the case that any given property instantiated by a proper part of the actual world is instantiated in virtue of a single property that is not independent of accompaniment (one like D), so pluralism is consistent with the in-virtue-of account's saying that some properties of proper parts of the world are instantiated in an intrinsic fashion while others are not.

\section{B. Modifying the Account}

So it looks like monism is incompatible with the in-virtue-of account. What now? Let us attempt to modify the in-virtue-of account in such a way that it retains its core insight and thus its original plausibility, but is compatible with both monism and pluralism. First I suggest that we, following Lewis in his first account of intrinsicality, just stipulate that the fundamental properties, whatever they turn out to be, are intrinsic. I do not think this stipulation is particularly regrettable given our earlier discussion of the intrinsic nature of fundamentality. With this stipulation, we can modify the in-virtue-of account thus:

Intrinsic fashion: $\mathrm{x}$ has $\mathrm{P}$ in an intrinsic fashion just in case (i) $\mathrm{P}$ is independent of accompaniment; and (ii) for any individual $\mathrm{y}$ and property $\mathrm{Q}$, if $\mathrm{x}$ has $\mathrm{P}$ in virtue of y's having $Q$, then $Q$ is either fundamental or independent of accompaniment. 
Intrinsicality: Property $\mathrm{P}$ is intrinsic just in case $\mathrm{P}$ is fundamental or, for any possible individual $\mathrm{x}$, if $\mathrm{x}$ has $\mathrm{P}, \mathrm{x}$ has $\mathrm{P}$ in an intrinsic fashion.

Let us call this the 'modified account'. Is it compatible with monism in addition to pluralism? Well, the modified account is not compatible with monism and pluralism in the sense that the account generates the same truths about intrinsicality independently of how the debate between the monist and pluralist turns out. This is so because on the modified account the pluralist would presumably claim that $\mathrm{D}$ (the actual global distributional property discussed earlier) is non-intrinsic, while the monist would claim that $\mathrm{D}$ is intrinsic given that it is fundamental. It is compatible with monism and pluralism, however, in the sense we care about: it can be both true and complete (in the sense of 'complete' discussed at the outset of this paper), and neither the monist nor the pluralist would thereby be committed to saying either that properties which intuition clearly dictates are intrinsic are really non-intrinsic or vice versa. With respect to monism, recall that the locus of the incompatibility of the original in-virtue-of account with monism is that the monist wants to say that $\mathrm{D}$, qua fundamental property, is intrinsic, but if the in-virtue-of account is true the monist would have to say that $\mathrm{D}$ is non-intrinsic, given that $\mathrm{D}$ is not independent of accompaniment. On the modified account, however, D is counted as intrinsic.

Even though the modified account is compatible with monism in the sense specified above, I can think of at least one reason why the monist should not endorse it. Though it is intuitive to think that non-intrinsic properties are instantiated ultimately in virtue of intrinsic properties, both the pluralist and the monist owe us a story about how exactly this is supposed to work; they both need to say something about how it is that the non-intrinsic features of the world are rooted in the intrinsic ones. Call this the 'origins of non-intrinsic properties' question. The pluralist has a straightforward and illuminating answer: various combinations of intrinsic properties instantiated at the fundamental level of reality - the level of atoms and, recalling our earlier discussion of external relations, certain fusions of them-are that ultimately in virtue of which non-intrinsic properties are instantiated at various levels of higher aggregation. The monist, on the other hand, claims that a single intrinsic property (D) instantiated at the fundamental level of reality - the level of the world as a whole - is that ultimately in virtue of which non-intrinsic properties are instantiated at various levels of decomposition, levels decomposed with respect to the world as a whole. The latter answer, however, is not as illuminating as the former; we can readily understand how it could be that the instantiation of non-intrinsic properties is to be accounted for by combinations of intrinsic properties and relations instantiated at levels of lesser aggregation, but this is not so for the claim that the instantiation of non-intrinsic properties is to be accounted for by the instantiation of a single intrinsic property at the level of least (or rather no) decomposition, the level of the world as a whole. The latter claim, if true, would seem to constitute a brute fact, and the positing of brute facts is, of course, ceteris 
paribus, to be avoided if possible. I therefore tentatively conclude that, though the modified account may be compatible with monism, the monist nevertheless should not endorse it.

\section{A Further Modification}

With a further modification to the modified account, again one that preserves the central insight behind the original account, we will have an account of intrinsicality that is not only compatible but also amenable to the pluralist and monist alike. This modification, I maintain, makes it possible for the monist, as well as the pluralist, to have a satisfactory answer to the origin of non-intrinsic properties question.

The modification I have in mind appeals to a distinction between two types of in-virtue-of relations, what I call the 'intra-virtue-of' relation and the 'inter-virtue-of' relation. The distinction is inspired by Jaegwon Kim's distinction between what he calls 'levels' and 'orders'. Kim writes:

I think we might usefully distinguish between 'higher-level' and 'higher-order,' or 'levels' and 'orders,' when speaking of properties in an ordering, using the 'order' idiom for first-order, second-order, third-order, ... properties, and reserving the 'level' idiom for tracking the micro-macro hierarchy. ... [The progression of orders] does not track the micro-macro ordering: these properties are all properties applying to entities at a single micro-macro level.

[1998: 83]

For Kim 'micro-macro' levels are mereological levels, what the monist is wont to call levels of 'decomposition' of the world, and the pluralist levels of 'aggregation'. But what is a mereological level? As Kim [2002] points out, the idea that there are levels of reality and that they have something to do with the part-whole relation goes back at least as far as C. Lloyd Morgan's Emergent Evolution [1923] and culminates in Oppenheim and Putnam's famous 1958 paper. I have the space neither to consider their proposals here nor properly motivate an account of mereological levels. ${ }^{27}$ So I will instead just state the conception I propose to work with. Consider the following figure illustrating the three-atom model of classical mereology:

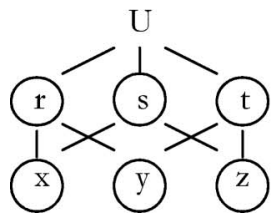

Level 3, the universe: $\mathrm{U}=\mathrm{x}+\mathrm{y}+\mathrm{z}$

Level 2, the molecules: $r=x+y ; s=x+z ; t=y+z$

Level 1, the atoms: $x, y$, and $z$

\footnotetext{
${ }^{27}$ I will say, however, that I find Kim's [2002] objections to their conceptions of levels persuasive.
} 
In this universe, there are three mereological levels: the level of atoms, that of two-atom molecules, and the world itself, a three-atom molecule. ${ }^{28}$ Hence, on the assumption that there are mereological atoms and they are finite in number, we can say that two individuals, $x$ and $y$, occupy the same mereological level only if they are composed of the same number of atoms. If, e.g., $\mathrm{x}$ is composed of more atoms than $\mathrm{y}, \mathrm{x}$ is on a higher level than $\mathrm{y}, \mathrm{a}$ level of less decomposition and one of greater aggregation. ${ }^{29}$

Kim claims both that $\mathrm{n}$-order properties realize $\mathrm{n}+1$ order properties, and that realization is an intra-level phenomenon, in the sense that a property $\mathrm{P}$ of an object $\mathrm{x}$ realizes property $\mathrm{Q}$ of an object $\mathrm{y}$ only if $\mathrm{x}$ and $\mathrm{y}$ occupy the same mereological level. What I call the 'intra-virtue-of' relation is modelled after Kim's conception of realization: $\mathrm{x}$ has some property intravirtue-of $\mathrm{y}$ 's having some property only if $\mathrm{x}$ and $\mathrm{y}$ occupy the same level of aggregation/decomposition of the world. Put another way, the intra-virtueof relation is ontological dependence between properties of objects that occupy the same mereological level. We can say, then, that realization just is the intra-virtue-of relation, or perhaps a species of it. ${ }^{30}$ What I call the 'inter-virtue-of' relation, therefore, is just this: $\mathrm{x}$ has some property intervirtue-of $\mathrm{y}$ 's having some property only if $\mathrm{x}$ and $\mathrm{y}$ occupy different levels of aggregation/decomposition of the world. Alternatively, the inter-virtue-of relation is ontological dependence between properties of objects that occupy different mereological levels. ${ }^{31}$

With the distinction between intra-virtue-of and inter-virtue-of relations on the table, we can say that when the pluralist claims that a property of the world is determined by various properties of its proper parts, she is claiming

\footnotetext{
${ }^{28}$ The figure above is adapted from Schaffer [forthcoming]. This is not, however, to say that Schaffer endorses my conception of mereological levels; he uses the figure for different purposes.

${ }^{29}$ Thanks to Schaffer for helpful discussion here. This conception of levels is, of course, much more finegrained than either Morgan or Oppenheim and Putnam (or Kim for that matter) have in mind; on this conception of levels it is not the case, e.g., that each of the sciences corresponds to a specific level. Earlier, in arguing that fundamental properties are intrinsic, I claimed that the pluralist is free to claim that the fundamental level of reality consists of mereological atoms as well as certain fusions of them, and the fundamental properties and relations are instantiated by the occupants of this level. On the way I have proposed to individuate levels of reality, in this case the pluralist would be claiming that the fundamental level consists of more than one mereological level.

${ }^{30}$ The determinable-determinate relation is also an intra-level in-virtue-of relation, so if it is distinct from the realization relation, it looks like we have at least two sorts of intra-virtue-of relations.

${ }^{31}$ Interestingly, Kim may reject the idea that there is something like the inter-virtue-of relation. Here is why. The inter-virtue-of relation requires so-called mereological supervenience, where the latter is understood either as properties of wholes supervening on properties of their parts, or vice versa. Kim claims that when we talk about mereological supervenience 'we often speak loosely', for what we mean to say is the 'base property on which $\mathrm{P}$ supervenes is ... the property of having such-and-such proper parts that have such-and-such properties and are configured by such-and-such relations', but this is 'not a property that belongs to any of its proper parts' [1998: 86]. He therefore concludes that 'in general' supervenient properties and their base properties are instantiated by objects at the same mereological level. If there is no genuine inter-level mereological supervenience, then it follows that the inter-virtue-of relation is not instantiated.

I agree that, for any claim about properties of wholes supervening on properties of parts or vice versa, there is a corresponding claim to be made about intra-level supervenience along the lines Kim proposes above. The availability of the latter claim, however, does not undermine the legitimacy of the former. Not only does it seem that the properties of wholes supervene on the properties of parts, but, as Schaffer [forthcoming] has shown, the properties of parts supervene on the properties of wholes as well. (Schaffer's claim is actually restricted to the supervenience of properties of proper parts of worlds on global properties, but it extends to the more general claim.) Perhaps Kim thinks that since every inter-level supervenience claim has a corresponding intra-level supervenience claim, we are forced to choose between them, and since realization is an intra-level relation and realization requires supervenience, we are to conclude that there is intra-level but no inter-level supervenience. If there were a scarcity of grounding properties, this thought might be well motivated, but, recalling our discussion of being a rock, it is unclear why we should not accept multiple, overlapping grounding properties.
} 
that the world has its property inter-virtue-of those parts' having their various properties. The same considerations apply to the monist; when the monist says that various properties of various proper parts of the world are determined by some property the world instantiates, she is claiming that those parts have those properties inter-virtue-of the world's having some property.

The monist and pluralist need not disagree about the intra-virtue-of facts, but they do disagree about the inter-virtue-of facts. The monist claims that, for any object $\mathrm{x}$ and $\mathrm{y}$, if $\mathrm{x}$ has some property inter-virtue-of $\mathrm{y}$ 's having some property, then $\mathrm{x}$ is a proper part of $\mathrm{y}$, while the pluralist claims that if $\mathrm{x}$ has some property inter-virtue-of y's having some property, then $\mathrm{y}$ is a proper part of $x$. Let me say a bit more about this claim. Suppose that Shoemakerstyle (rather than Lewis-style) functionalism is true, and consider, e.g., the mental property $\mathbf{M}$, such that $\mathbf{M}$ is the property of having some property or other, instances of which play causal role R. Suppose that physical property $\mathrm{P}$ is instantiated and P-instances play role $\mathrm{R}$. As I understand monism, pluralism, and the distinction between inter-level and intra-level in-virtue-of relations, the monist will say that $M$ is instantiated intra-virtue-of the instantiation of $\mathrm{P}$, and $\mathrm{M}$ (and $\mathrm{P}$ ) is instantiated inter-virtue-of, among other, things, the world's instantiating D. Though the pluralist, I claim, will agree that $\mathrm{M}$ is instantiated intra-virtue-of $\mathrm{P}$, she will claim that $\mathrm{M}$ (and $\mathrm{P}$ ) is instantiated inter-virtue-of certain properties of certain objects at various levels of reality of lesser aggregation than the level occupied by the object that instantiates M. (One and the same property, then, can be instantiated intra-virtue-of and inter-virtue-of different properties.) My claim is that the disagreement between the pluralist and the monist, then, is only about the mereological direction of the inter-virtue-of relations; one claims they go from part to whole, the other from whole to part. ${ }^{32}$

Since the monist and the pluralist need not disagree about the intravirtue-of facts, let us recast the modified account in terms of the intra-virtueof relation, but again in such a way as to preserve the core insight of the original account:

Intrinsic fashion: $\mathrm{x}$ has $\mathrm{P}$ in an intrinsic fashion just in case (i) $\mathrm{P}$ is independent of accompaniment; and (ii) for any individual $\mathrm{y}$ and property $\mathrm{Q}$, if $\mathrm{x}$ has $\mathrm{P}$ intra-virtue-of y's having $\mathrm{Q}$, then $\mathrm{Q}$ is either fundamental or independent of accompaniment.

\footnotetext{
${ }^{32}$ Having drawn the distinction between inter-level and intra-level in-virtue-of relations, we can say that $\mathrm{P}$ is a fundamental property only if it is necessary that, for any object $\mathrm{x}$, if $\mathrm{x}$ has $\mathrm{P}$, there is no object $\mathrm{y}$ and property $\mathrm{Q}$ such that $\mathrm{x}$ has $\mathrm{P}$ either inter-virtue-of or intra-virtue-of $y$ 's having $\mathrm{Q}$. The norm for properties is that they are instantiated both intra-virtue-of certain properties and inter-virtue-of certain properties. Fundamental properties, of course, are not like this, but there are certain non-fundamental properties that are not like this either. Suppose that monism is true and the world instantiates property $\mathrm{P}$ such that $\mathrm{P}$ and $\mathrm{D}$ are distinct. Assuming that D is the only fundamental property, $\mathrm{P}$ is a non-fundamental global property, such that $\mathrm{P}$ is instantiated intra-virtue-of $\mathrm{D}$, though $\mathrm{P}$ is not instantiated inter-virtue-of any property. (The determinables of D we considered earlier in responding to Caplan's objection are like P.) Now suppose instead that pluralism is true. If a property $\mathrm{P}$ is instantiated by a mereological atom but $\mathrm{P}$ is not fundamental, then, though $\mathrm{P}$ is not instantiated inter-virtue-of any property, $\mathrm{P}$ is instantiated intra-virtue of some fundamental property of the atom. Returning to the matter of non-qualitative properties like being identical to Kelly (see note 6), it seems that these properties are non-fundamental and instantiated neither intra-virtue-of nor intervirtue-of any properties.
} 
Intrinsicality: Property $\mathrm{P}$ is an intrinsic property just in case $\mathrm{P}$ is fundamental or, for any possible individual $\mathrm{x}$, if $\mathrm{x}$ has $\mathrm{P}, \mathrm{x}$ has $\mathrm{P}$ in an intrinsic fashion.

Call this the 'further modified account'. Before considering this account, let us briefly review how our conception of intrinsicality has evolved over the paper. On the original in-virtue-of account, $\mathrm{P}$ is intrinsic just in case it is independent of accompaniment, and for any $\mathrm{Q}$, if $\mathrm{P}$ can be instantiated in virtue of $\mathrm{Q}, \mathrm{Q}$ itself is independent of accompaniment. The modified account embodies a refinement concerning fundamental properties: $\mathrm{P}$ is intrinsic just in case $\mathrm{P}$ is fundamental or independent of accompaniment, and, for any $\mathrm{Q}$, if $\mathrm{P}$ can be instantiated in virtue of $\mathrm{Q}, \mathrm{Q}$ is either independent of accompaniment or fundamental. In the further modified account, we have a refinement concerning the intra-virtue-of relation: $\mathrm{P}$ is intrinsic just in case $\mathrm{P}$ is fundamental or independent of accompaniment, and, for any $\mathrm{Q}$, if $\mathrm{P}$ can be instantiated intra-virtue-of $\mathrm{Q}, \mathrm{Q}$ is either independent of accompaniment or fundamental.

Let us consider an example to illustrate how the account works with its restriction to intra-virtue-of relations. Consider in particular another example of a property that is independent of accompaniment yet intuitively non-intrinsic, this time one proposed by John Hawthorne [2001], being such that there is something to which one is attending. What we want is a possible case in which this property is instantiated in a non-intrinsic fashion as specified by the further modified account. Suppose that in the actual world Frank instantiates this property intra-virtue-of attending to himself. (The relevant in-virtue-of relation is intra-level rather than inter-level because Frank, of course, occupies the same mereological level as himself.) The property attending to one's self is independent of accompaniment, so on this occasion being such that there is something to which one is attending is instantiated in an intrinsic fashion. This, therefore, is not the case we are looking for. There is a possible world w, however, in which Frank attends to a statue that is composed of the same number of mereological atoms he is composed of. ${ }^{33}$ Hence, in $\mathrm{w}$, Frank instantiates being such that there is something to which one is attending in virtue of Frank and the statue jointly instantiating the relation $\mathrm{x}$ is attending to a wholly distinct $\mathrm{y}$. This relation is instantiated by individuals (Frank and the statue) on the same mereological level as the individual who instantiates being such that there is something to which one is attending (Frank), so on this occasion the latter property is instantiated intra-virtue-of the former. Since the former property is neither independent of accompaniment nor fundamental, we have the case we have been looking for: a possible case in which our target property is instantiated in a non-intrinsic fashion. This reasoning is available to the monist as well as the pluralist. Hence, on either thesis the further modified account classifies the property as non-intrinsic. The consideration of further examples would

\footnotetext{
${ }^{33}$ Perhaps all the statues in the actual world Frank ever has attended to, and ever will, are composed of different numbers of mereological atoms than Frank. Depending on how we think about the conditions for composition, it may even turn out that Frank is the only actual individual to be composed of such-and-such number of atoms, past, present, and future. But surely there are merely possible worlds in which the relevant statue and Frank occupy the same mereological level.
} 
reveal that the further modified account is compatible with monism and pluralism in the same sense that the modified account is. ${ }^{34}$

Now we can return to the origin of non-intrinsic properties question. Consider all the non-intrinsic properties and relations instantiated by objects that occupy mereological level 1 , where 1 falls somewhere in between the level of the world as a whole and the level of mereological atoms. I have argued that, with the modified account, the pluralist but not the monist has an illuminating explanation of how the non-intrinsic features instantiated by objects on 1 are grounded in intrinsic properties and relations. Again, the idea is that the pluralist can claim that these features are grounded in the instantiation of various combinations of fundamental intrinsic features, while the monist can only say that they are grounded in the instantiation of a single intrinsic fundamental global distributional property, D. But if the further modified account is true, the monist and pluralist both have illuminating answers to the origins of non-intrinsic properties question, and, interestingly, they have the same answer. The answer proceeds as follows.

The non-intrinsic features on 1 are not grounded in the relevant sense by way of the instantiation of intrinsic properties and relations by objects on some other mereological level. Instead, the non-intrinsic features on 1 are grounded in the relevant sense by the intrinsic features of objects on the very same mereological level. Why think that this is so? Well, the further modified account says that the grounding relations relevant to whether a property is intrinsic or not are all instantiated at the same mereological level as the target property itself, so when we talk about how non-intrinsic features are supposed to be grounded in intrinsic ones, on the further modified account it is a mistake to focus on grounding relations the instantiation of which involves movement across mereological levels. Instead, the objects that instantiate the intrinsic features that ground the non-intrinsic features under consideration occupy the very same mereological level as those objects that have the relevant non-intrinsic features. This answer to the origins of nonintrinsic properties question, given its restriction to matters intra-level, is neutral between monism and pluralism. Finally it seems that we have an account of intrinsicality amenable to both the monist and the pluralist. Moreover, given that the further modified account preserves the core insight of the original in-virtue-of account, it is independently plausible.

\section{An Objection}

Sider [personal communication] has suggested something like the following objection to my response to the objection from intrinsicality to monism. ${ }^{35}$ Consider the property being a part of a city. Sider has suggested that this

\footnotetext{
${ }^{34}$ For another example, let us return to being cubical and lonely or non-cubical and accompanied. Consider a possible world $\mathrm{w}$ in which an individual (a shoe) instantiates this property in virtue of the shoe and, say, a book standing in the relation $\mathrm{x}$ (the shoe) is next to a wholly distinct $\mathrm{y}$ (the book), which, of course, is not independent of accompaniment. Supposing that the shoe and the book occupy the same mereological level in $\mathrm{w}$, the disjunctive property is instantiated intra-virtue-of the instantiation of the relation, so the former is classified as non-intrinsic.

${ }^{35}$ Frank Scott [personal communication] has posed a related objection.
} 
property is a disjunctive property, being (identical to) a city or being a proper part of a city, and let us grant this assumption. The disjunctive property is intuitively non-intrinsic, ${ }^{36}$ but it is independent of accompaniment, for it is possible that a lonely object has it (a lonely city), a lonely object lacks it (a lonely sphere), an accompanied object has it (a bridge that is a proper part of a city), and an accompanied object lacks it (a sphere in a world that is accompanied by other objects but no cities). Sider suggests that the further modified account may (wrongly) classify the disjunctive property as intrinsic.

According to the account of intrinsicality we are working with, since the disjunctive property is independent of accompaniment and non-fundamental, it is non-intrinsic just in case there is some property $\mathrm{P}$ that meets the following two conditions: (i) it is possible for the disjunctive property to be instantiated intra-virtue-of $\mathrm{P}$; and (ii) $\mathrm{P}$ is not independent of accompaniment. The disjunctive property can be instantiated in virtue of either of its disjuncts, being (identical to) a city or being a proper part of a city. Neither property, however, is a plausible candidate for a P-property, for it seems that the former meets condition (i) but not (ii), while the latter meets condition (ii) but not (i). It is clearly possible for the disjunctive property to be instantiated intra-virtue-of being (identical to) a city, but it seems that the latter is independent of accompaniment. Turning to being a proper part of a city, suppose that a bridge instantiates the disjunctive property in virtue of its instantiation. For an object $\mathrm{x}$ to be a proper part of an object $\mathrm{y}$ is for $\mathrm{x}$ and $\mathrm{y}$ to instantiate a certain relation, and $\mathrm{x}$ and $\mathrm{y}$ are so related only if they occupy different mereological levels. Hence, if the bridge instantiates being $a$ part of a city in virtue of being a proper part of a city, though the latter is not independent of accompaniment, ${ }^{37}$ the bridge instantiates the former intervirtue-of the latter. If the two properties just discussed are the only plausible candidates for P-properties, then it looks like the further modified account misclassifies the disjunctive property as intrinsic.

Here is perhaps a more intuitive way to put the objection. As we have seen, the further modified account says that the grounding relations relevant to whether being a part of a city is intrinsic or not are all instantiated by possible individuals that occupy the same mereological level as the individual that instantiates the disjunctive property. Sider's objection proceeds upon the idea that the only possible grounding relation for being a part of a city to 'go non-intrinsic by' is an inter-level grounding relation, that of being instantiated inter-virtue-of being a proper part of a city. The idea is that if we are limited to considering in-virtue-of relations instantiated by objects at the same mereological level as the bridge, we are forced to conclude that being a part of a city is intrinsic. The objection, then, is that, given the restriction to intra-level grounding relations, the further modified account is too strong.

\footnotetext{
${ }^{36}$ The property being a city, I take it, is a paradigmatic 'border-sensitive' property in Sider's [2001] sense.

${ }^{37}$ Why is being a proper part of city not independent of accompaniment? Well, if an object $\mathrm{x}$ is a proper part of $\mathrm{y}$, then there is some $\mathrm{z}$ wholly distinct from $\mathrm{x}$ that is also a proper part of $\mathrm{y}$. Hence, a lonely object cannot instantiate being a proper part of a city.
} 
What should we make of this objection? My response is straightforward: there are other promising candidates for P-properties; in other words, there are possible intra-level grounding relations for being a part of a city to go non-intrinsic by. If the bridge is a proper part of the city, then it is possible for there to be objects on the same mereological level the bridge occupies that are also proper parts of the city. Consider a possible world $\mathrm{w}$ in which, say, a museum and the sum of all parked Volvos occupy the mereological level occupied by the bridge, i.e. they are all composed of the same number of mereological atoms. Suppose further that the museum and the sum of Volvos are also proper parts of the city in question in $\mathrm{w}$. Now consider the property being accompanied by a museum and a sum of parked Volvos. I submit that if the bridge instantiates being a part of a city inter-virtue-of the bridge and the city jointly instantiating $\mathrm{x}$ (the bridge) is a proper part of $\mathrm{y}$ (the city) in $\mathrm{w}$, then the bridge instantiates the former and latter properties in virtue of the museum/Volvo property in w as well. Why think that this is so? In general, what makes an individual a proper part of a whole is that the individual is a part of the whole, and it is accompanied by wholly distinct objects that are also parts of the whole. In $\mathrm{w}$, what makes the bridge a proper part of the city is that it is a part of the city, and there are wholly distinct objects on the same mereological level, namely the museum and the sum of parked Volvos, that are also parts of the city. Given that (trivially) the bridge is on the same mereological level as itself, the bridge instantiates being a part of a city intra-virtue-of its instantiating the museum/Volvo property. The latter property clearly is not independent of accompaniment, so it meets both conditions (i) and (ii) for being a P-property. In other words, it looks as if we have found an intra-level grounding relation for the disjunctive property to go non-intrinsic by: being a part of a city is non-intrinsic because there is a possible world in which it is instantiated in virtue of a property instantiated at the same mereological level that is not independent of accompaniment. Hence, it seems that, on the further modified account, being a part of a city is classified (correctly) as a non-intrinsic property.

\section{Conclusion}

In this paper I have responded to the objection from intrinsicality to monism (the claim that, since monism is incompatible with our best account of intrinsicality, monism is probably false) by proposing an account of intrinsicality that is amenable to both the monist and pluralist alike. Sider [2007] poses two other objections to monism, but to my mind the intrinsicality objection is, prima facie, the most troubling of the three. ${ }^{38} \mathrm{I}$ conclude that if there is a problem with monism, it must come from other quarters. Moreover, the significance of what I have done in this paper goes beyond merely responding to an objection to monism, for I have proposed

\footnotetext{
${ }^{38}$ Schaffer [unpublished $\mathrm{ms}$ ] agrees with this assessment.
} 


\title{
an account of intrinsicality that is not only neutral between monism and pluralism but is also independently plausible. ${ }^{39}$
}

\author{
University of Massachusetts-Amherst
}

\author{
Received: April 2007 \\ Revised: July 2007
}

\section{References}

Hawthorne, John 2001. Intrinsic Properties and Natural Relations, Philosophy and Phenomenological Research 63/2: 399-403.

Kim, Jaegwon 1998. Mind in a Physical World, Cambridge, Mass.: MIT Press.

Kim, Jaegwon 2002. The Layered Model: Metaphysical Considerations, Philosophical Explorations 5: 2-20.

Langton, Rae and David Lewis 1998. Defining Intrinsic, Philosophy and Phenomenological Research 58/2: 333-45.

Leibniz, G. W. 1975 (1702). Leibniz to deVolder, April 1702, Philosophical Papers and Letters, $2^{\text {nd }}$ edition, ed. L. E. Loemker, New York: Springer.

Lewis, David 1983. New Work for a Theory of Universals, Australasian Journal of Philosophy 61: 343-77.

Lewis, David 1986. On the Plurality of Worlds, Oxford: Basil Blackwell.

Lewis, David 2001. Redefining Intrinsic, Philosophy and Phenomenological Research 63/2: 381-91.

Morgan, C. Lloyd 1923. Emergent Evolution, New York: Henry Holt and Co.

Oppenheim, Paul and Hilary Putnam 1958. Unity of Science as a Working Hypothesis, in Minnesota Studies in the Philosophy of Science, Vol. 2, ed. H. Feigl, M. Scriven, and G. Maxwell, Minneapolis: University of Minnesota Press: 3-36.

Parsons, Josh 2004. Distributional Properties, in Lewisian Themes, ed. F. Jackson and P. Graham, Oxford: Oxford University Press: 173-80.

Pereboom, Derk unpublished ms. Physicalism and Absolutely Intrinsic Properties. URL $=\mathrm{http}: / /$ consciousness.anu.edu.au/events/revelation.html.

Schaffer, Jonathan 2005. Quiddistic Knowledge, Philosophical Studies 123: 1-32.

Schaffer, Jonathan 2007a. Monism, The Stanford Encyclopedia of Philosophy (Summer 2007 Edition), ed. Edward N. Zalta, URL = http://plato.stanford.edu/archives/sum2007/entries/monism/.

Schaffer, Jonathan 2007b. From Nihilism to Monism, Australasian Journal of Philosophy 8/2: 175-91.

Schaffer, Jonathan unpublished ms. For (Priority) Monism: A Reply to Sider. URL=http://people. umass.edu/schaffer/papers/ReplySider.pdf.

Schaffer, Jonathan Forthcoming. Monism: The Priority of the Whole, Philosophical Review, URL $=$ http:// people.umass.edu/schaffer/papers/Monism.pdf.

Sider, Ted 1993. Naturalness, Intrinsicality, and Duplication, $\mathrm{PhD}$ thesis. URL $=$ http://fas-philosophy. rutgers.edu/sider/papers/diss.pdf.

Sider, Ted 1996. Intrinsic Properties, Philosophical Studies 83: 1-27.

Sider, Ted 2001. Maximality and Intrinsic Properties, Philosophy and Phenomenological Research 63/2: 35763.

Sider, Ted 2007. Against Monism, Analysis 67/1: 1-7.

Sider, Ted unpublished ms. Monism and Statespace Structure. URL $=$ http://homepages.nyu.edu/ $\sim$ ts65/.

Vallentyne, Peter 1997. Intrinsic Properties Defined, Philosophical Studies 88: 209-19.

Weatherson, Brian 2001. Intrinsic Properties and Combinatorial Principles, Philosophy and Phenomenological Research 63/2: 365-80.

Weatherson, Brian 2006. The Asymmetric Magnets Problem, Philosophical Perspectives 20: 479-92.

Witmer, D. Gene, Bill Butchard, and Kelly Trogdon 2005. Intrinsicality without Naturalness, Philosophy and Phenomenological Research 70: 326-50.

Yablo, Steve 1998. Intrinsicness, Philosophical Topics 26: 479-504.

\footnotetext{
${ }^{39}$ In an unpublished manuscript made available after the completion of this paper, Sider proposes his own solution to the objection from intrinsicality on behalf of the monist, though he argues that the monist still faces other problems. (He hints at this response in Sider [2007: $6 \mathrm{n}$.14]. Sider's response is complex and, as of now (late November 2007), still in the works, so I won't consider it here. I would like to thank Phil Bricker, Ben Caplan, Maya Eddon, Timmy Fuller, Frank Scott, Ted Sider, Nick Treanor, Brian Weatherson, Gene Witmer, and two anonymous referees from the AJP for helpful comments and discussion. I especially wish to thank Jonathan Schaffer for bringing monism to my attention (and convincing me that it is worth taking seriously!), as well as for many helpful comments on this paper.
} 